Artigos/Articles

\title{
Construcciones de voz media y alternancias de la transitividad en lengua toba (Flia. Guaycurú)
}

Construções da voz média e alternâncias da transitividade na língua toba (Flia. Guaicuru)

Middle constructions and transitivity alternations in Toba language (Guaycuruan Family)

Adriana Alicia Zurlo ${ }^{1,2}$

\section{RESUMEN}

Este artículo analiza desde una perspectiva tipológico-funcional construcciones de voz media en lengua toba (familia guaycurú). A diferencia de otros estudios previos, se argumenta que la voz media, expresada a través del paradigma verbal n-, muestra características típicas de un sistema de voz básica activa-media (Klaiman 1991). El objetivo es describir, dentro del continuo heterogéneo de construcciones medias alternantes del toba, varias operaciones de ajuste de valencia o alternancias de la transitividad con morfemas verbales aplicativos, el sufijo reflexivo (-la?t) y el sufijo reciproco (-a?t). La voz media en esta

1. Instituto de Investigaciones Geohistóricas, dependiente del Consejo Nacional de Investigaciones Científicas y Técnicas (CONICET) y de la Universidad Nacional del Nordeste (UNNE). Ciudad de Resistencia, Provincia de Chaco - Argentina. https://orcid. org/0000-0002-1098-9807. E-mail: adrianazurlo@hum.unne.edu.ar.

2. Consejo Nacional de Investigaciones Científicas y Técnicas (CONICET). 
lengua es una categoría semántica compleja que no siempre involucra detransitivización semántico-sintáctica.

Palabras clave: voz media; transitividad; valencia verbal; tipología funcional.

\title{
RESUMO
}

Em este trabalho, analisam-se construções da voz média na língua toba (família Guaicuru), seguindo uma perspectiva tipológico-funcional. Ao contrário de estudos prévios, explica-se aqui que a voz média é expressa com o paradigma flexional verbal n e mostra características típicas de um sistema de voz básica ativa-média (Klaiman 1991). O objetivo deste estudo é descrever dentro do continuo heterogêneo de construções médias na língua toba, várias alternâncias de transitividade tais como aplicativos, reflexivo (-la?t) e recíproco (-a?t), que co-ocorrem com verbos médios alternados. A voz média nesta língua é uma categoria semântica complexa que nem sempre envolve a destransitivação semântico-sintática.

Palavras-chave: voz média; transitividade; valência verbal; tipologia funcional.

\begin{abstract}
This article analyzes middle constructions in toba language (Guaycuruan family) from a typological and functional approach. It is argued here that middle voice is coded with verbal paradigm n- and shows typical characteristics of a basic voice system (Klaiman 1991). The aim of this study is to describe within the heterogeneous continuum of middle constructions in this language, several transitivity alternations such as applicatives, reflexive (-la?t), reciprocal (-a?t), which coocurres with middle alternates verbs. The middle voice in this language is a complex semantic category that does not only involve semantic-syntactic detransitivization.
\end{abstract}

Keywords: Middle voice; transitivity; verbal valence; functional tipology. 


\section{Introducción}

El objetivo de este artículo es analizar construcciones de voz media en la lengua toba y, en particular, describir algunas alternancias de la transitividad que ocurren junto a verbos medios alternantes.

El trabajo aborda el estudio de construcciones con el paradigma de índices pronominales o prefijos verbales $n$-, el cual ha sido objeto de múltiples y no siempre coincidentes análisis entre los estudios previos referidos al toba y a otras lenguas guaycurúes. Con relación al toba, se caracteriza formalmente el prefijo verbal $n$ - como parte de un paradigma flexivo, como un indicador del actor y de la orientación de la acción (Buckwalter y Buckwalter 2004, Bigot 1994), como reflexivo (Buckwalter y Buckwalter 2004), como marca de acción adcorpórea (Klein 1981), como amalgama de voz media, persona y número (Censabella 1998, 2002), de agente afectado e integrando un paradigma de caso activo, en oposición al paradigma inactivo o pacientivo (Messineo 2002, 2003). Por otro lado, también con relación a otras lenguas guaycurúes se discute el estatuto del prefijo verbal $n$-, que ha sido analizado como parte de un sistema de marcación de caso agentivo-pacientivo (Vidal 2001, para el pilagá), como marca del agente afectado en un sistema activo-inactivo (Gualdieri 1998, para el mocoví), como un marcador reflexivo y de 'hither' (Grondona 1998, para el mocoví).

Estas diferentes interpretaciones permiten vislumbrar algunas problemáticas teórico-analíticas en torno a los índices pronominales del verbo toba, una discusión que no se ha resuelto aún. En este sentido, este artículo pretende sumar un nuevo análisis sobre el paradigma verbal $n$ - en dicha lengua.

Partimos del estudio de Censabella (1998 y 2002), quien plantea que la voz media en toba se expresa a través del paradigma verbal $n$ - e indica la afectación del sujeto/agente en la acción y, planteamos además, que la voz media en toba exhibe características de un sistema de voz básica, tal como lo describe Klaiman (1991) a partir de ejemplos de sánscrito y griego clásico, fula y tamil.

Según nuestra hipótesis (Zurlo 2016a/b), la voz media en toba manifiesta características de un sistema de voz básica ya que 
organiza el lexicón verbal en tres clases, a partir de sus marcas de voz: verbos activos invariantes, medios invariantes y alternantes. La voz media comprende construcciones heterogéneas: raíces verbales invariantes (media léxica) y una mayoría de raíces alternantes (media morfológica). En el grupo de verbos alternantes, la voz media se asocia a numerosas alternancias de la transitividad que no siempre manifiestan detransitivización semántico-sintáctica (pérdida argumental). Por otro lado, el objetivo específico de este artículo es mostrar construcciones medias alternantes combinadas con sufijos verbales que aumentan (aplicativos) y otros que típicamente disminuyen o reordenan la transitividad (reflexivo y recíproco).

El corpus analizado comprende texto oral recopilado mediante entrevistas y datos elicitados con hablantes de referencia (lexemas, sintagmas verbales y nominales, paradigmas de conjugación y cláusulas). Se complementa con textos publicados de género narrativo. De manera permanente hemos consultado el Vocabulario toba de A. Buckwalter y L. Buckwalter [2004 (1980)].

El trabajo se organiza en los siguientes apartados: $\S 1$, donde presentamos brevemente características de la lengua toba o qom l7aqtaqa; en $\$ 2$, aspectos teóricos referidos a voz media y sistema de voz básica; en $\S 3$, analizamos construcciones de voz media en toba, comenzando con los patrones de voz detectados en el lexicón verbal analizado ( $§ 3.1)$, seguido de las alternancias de la transitividad manifiestas junto a verbos medios alternantes (\$3.2) y finalizamos en $\S 4$ con las conclusiones.

\section{Características tipológicas de la lengua Toba o $Q O M$ LPAQTAQA}

El pueblo que se autodenomina qom, según datos censales, está compuesto por 126.967 personas en Argentina (INDEC 2012) y 2.057 en Paraguay (DGEEC 2013). En Argentina se nuclean en las provincias de Chaco y Formosa, en el este de Salta y como resultado de migraciones también en Rosario, Santa Fe y Buenos Aires.

La lengua toba pertenece a la familia lingüística guaycurú, integrada también por mbayá y y caduveo (guaycurú septentrional), mocoví, pilagá 
y abipón ${ }^{\dagger}$ (guaycurú meridional). Por su tipo morfológico, esta lengua se considera aglutinante, con tendencia a la polisíntesis y marcación de núcleo ('head-marking') en lo que respecta a la relación entre predicado y argumentos y en la frase nominal posesiva (Carpio 2007b). El orden de palabras más frecuente es VS, AVP y PVA cuando $\mathrm{P}^{3}$ es un pronombre libre. Esta lengua posee oposición verbo/nombre y no tiene marcas de caso, tampoco adjetivos como clase de palabras. Respecto del sintagma nominal, distingue nombres alienables e inalienables. Posee modificadores de género ('masculino' y 'femenino'), número nominal (singular no marcado y varios alomorfos de plural), prefijos posesivos (alienable/inalienable) y seis deícticos o determinantes demostrativos, cuyas unidades son: ra demostrativo 'parado', $\tilde{n} i$ 'demostrativo 'sentado', ze demostrativo 'acostado', na demostrativo de 'acercamiento', so demostrativo 'alejamiento', $k a$ demostrativo 'no presente'.También marca morfológicamente categorías afines al número, como distributivo y colectivo y expresa sintácticamente número dual (Carpio y Censabella 2010). Respecto de los verbos, el sistema de la conjugación está organizado en función de una oposición de voz activa/media y cuenta, por tanto, con dos paradigmas para todas las personas, el de voz activa y de voz media (tabla 1). Los verbos se clasifican, según el paradigma que toman, en: (i) activos; (ii) medios y (iii) alternantes. Respecto del alineamiento de los índices pronominales en los verbos, Carpio (2007b) observa las siguientes escisiones en la marcación de $\mathrm{S}, \mathrm{A}$ y $\mathrm{P}$, para la voz activa: $\left(1^{\circ}\right)$ una jerarquía de persona que distingue una marcación nominativo-acusativa para los participantes del acto de habla (1ra. y 2 da. personas); $\left(2^{\circ}\right)$ un sistema tripartito $(\mathrm{S} \neq \mathrm{A} \neq \mathrm{P})$ para los no-participantes del acto de habla (3ra. persona), es decir, un sistema en el que reciben diferentes marcas los argumentos S, A y P. Las raíces transitivas seleccionan el marcador de 3ra persona $i$ - [y-]. A su vez, S presenta cuatro formas distintas, que se encuentran en su gran mayoría lexicalizadas e indican distintos

3. Siguiendo la terminología propuesta por Comrie (1978:331), identificamos con la sigla S (single) al argumento único de una cláusula intransitiva, con la sigla A (agent) al argumento más parecido a un agente prototípico de una cláusula transitiva y con la sigla $\mathrm{P}$ (patient) al argumento más parecido a un paciente prototípico de una cláusula transitiva. En este sentido, el orden de constituyentes VS comprende el verbo seguido del argumento único (sujeto); el orden AVP, comprende un argumento central (sujeto), el verbo y un argumento central no sujeto (objeto) y, finalmente, el orden PVA, comprende un argumento central no sujeto (objeto) seguido del verbo y de un argumento central (sujeto). 
grados de control y afectación del participante único: $r$ - (intransitivo inergativo e inacusativo), $w$ - (intransitivo estativo), $\varnothing$ - (intransitivo de movimiento) y $t$ - (intransitivo traslacional). ( $\left.3^{\circ}\right)$ Para unas pocas raíces verbales, hay una manera diferente de codificar $\mathrm{P}$ cuando se trata de 1ra. singular (3i-), 2da. singular (?ar-) y 1ra. plural (qar-); este último subsistema sigue un patrón de marcación del tipo activo/ inactivo. La tabla 1 ilustra los paradigmas de prefijos personales o índices pronominales del verbo toba, que constituyen una amalgama de voz ${ }^{4}$, persona y número.

Tabla 1 - Paradigmas de índices pronominales o prefijos personales del verbo toba (Adaptado de Censabella 2002)

\begin{tabular}{|c|c|c|}
\hline $\begin{array}{l}\text { PERSONA } \\
\mathrm{y} \mathrm{N}^{\mathrm{o}}\end{array}$ & Paradigma I: voz activa & Paradigma II: voz media \\
\hline $1 \mathrm{SG}$ & $\mathrm{s}-$ & [in-] \\
\hline $1 \mathrm{PL}$ & $s-\ldots-q$ & {$[$ in-...-q] } \\
\hline $2 \mathrm{SG}$ & 2a-; Paw- & [an- $]$ \\
\hline $2 \mathrm{PL}$ & qaw...i & qan...i \\
\hline $3 \mathrm{SG}$ & i- [y-]; r- [d-]; Ø-; 1-; t-; w- & n- \\
\hline 3 PL & $\begin{array}{l}\mathrm{i}-\ldots-\mathrm{P}-[\mathrm{y}-\ldots \mathrm{P}] ; \mathrm{r}-\ldots .+\varnothing-\ldots \mathrm{\varnothing} ; 1-\ldots \mathrm{P} \\
\mathrm{t}-\ldots \mathrm{P} ; \mathrm{w}-\ldots \mathrm{P}\end{array}$ & $n-\ldots ?-$ \\
\hline
\end{tabular}

Por otro lado, la morfología verbal no expresa tiempo pero sí aspecto perfectivo (no marcado) e imperfectivo (subdividido en continuo -ta y progresivo -tak) y aspecto menor o número verbal, que presenta distintos matices según la raíz verbal con la que se combine (iterativo/intensificador - pek); además de modo desiderativo (-ayke) (Censabella 2002, 2008; Carpio 2007a/b; González 2013, Zurlo 2011, 2014). Finalmente, existen morfemas verbales muy productivos para codificar locación y dirección, se trata de: (i) un paradigma de sufijos direccionales y (ii) un paradigma de locativo-orientativos, que se comportan en su mayoría como aplicativos. La lengua permite relativizar sujetos y objetos y posee un patrón de coordinación de cláusulas nominativo-acusativo (pivote S/A) (Censabella 2002).

4. Entendida como categoría verbal flexiva que señala la orientación de la acción con relación al/los participante/s central/es (Benveniste 1966[1950]). 


\section{Aspectos teóricos}

\subsection{En torno a voz media y al sistema de voz básica}

Desde una perspectiva tipológica funcional, la voz media es definida por Kemmer (1993) como una categoría semántica compleja que comprende distintos tipos de situaciones; además, sintácticamente, las construcciones de voz media están más próximas al polo de la intransitividad que una construcción reflexiva prototípica.

Por otra parte, Klaiman (1991) plantea la existencia de un tipo particular de sistema de voz al que denomina voz básica a partir de la observación de patrones de marcación en el léxico verbal de lenguas diversas como sánscrito clásico, griego clásico (Indoeuropea), tamil (lengua dravídica del sur de la India) y fula (lengua nigerocongolesa). En un sistema de voz básica como el de voz activa/media en sánscrito clásico, ambas voces cuentan con morfología flexiva particular, como se ilustra en (1) con el verbo $k r$ - 'hacer, fabricar' que admite ambas voces $(-i /-e)^{5}$.

[Sánscrito clásico. Fuente: Klaiman 1991:24]
(1a) Devadattah
kațạ̣
karoti
Devadatta.NOM
mat.ACC
makes.3SG ACTIVE ${ }^{6}$
'Devadatta makes a mat.' /'Devadatta hace un tapete.'
(1b) Devadattah
Devadatta.NOM
kațạ
kurute
'Devadatta makes (himself) a mat.' /'Devadatta se hace un tapete.'

La autora observa que en ambos casos (1a, 1b), los argumentos presentan el mismo tipo de propiedades morfosintácticas y semánticas: (i) semánticamente Devadattạ̣ es el agente y kațm 'el tapete', el paciente; (ii) sintácticamente: Devadattaḥ presenta caso nominativo,

5. En este sentido, no hay una voz más básica que la otra y, por eso se argumenta que la oposición activa y media en estas lenguas es diferente a la oposición activa y pasiva perifrástica de las lenguas indoeuropeas, ya que esta última conformaría un sistema de voz derivada.

6. Glosa de la autora. Abreviaturas: $\mathrm{Nom}=$ nominativo; $\mathrm{ACC}=$ acusativo; $\mathrm{ACTIVE}=(\mathrm{voz})$ activa; INSTR=instrumental; $\mathrm{MIDDLE}=(\mathrm{voz})$ media; $\mathrm{PAST}=$ Pasado; $\mathrm{SG}=$ singular. 
rige la concordancia verbal y funciona como sujeto, mientras que katam 'el tapete' está marcado con caso acusativo y funciona como objeto.

Klaiman señala que en sánscrito clásico también es frecuente otro tipo de alternancia entre verbos que son transitivos en su forma activa e intransitivos en su forma media (2). Cuando ocurren con voz media (2b), se los usa intransitivamente para expresar acciones o eventos espontáneos. Esta función diferencial de la voz media llamada MEDIA NEUTRA (neuter middle), que tiende a ser incoativa, expresa un cambio de estado sin mencionar al agente o la causa pero no es una pasiva ${ }^{7}$ propiamente dicha.
(2a) Devadatto namati dandam devadatta.NOM bends.SG ACTIVE stick.ACC 'Devadatta bends the stick' / 'Devadata inclina la rama'
(2b) Namate dạ̣dah. bends.SG MIDDLE stick.NOM 'The stick bends' / 'La rama se inclina'

Además de los verbos alternantes, el sánscrito clásico cuenta con verbos invariantes, es decir, activa tantum y media tantum que se presentan en la tabla 2.

Tabla 2 - Verbos activa tantum y media tantum del sánscrito clásico (Klaiman 1991: 99)

\begin{tabular}{|l|l|}
\hline Activa tantum & Media tantum \\
\hline ad- 'eat/ comer' & labh- 'receive' 'recibir' \\
\hline as- 'be/ ser-estar' & $\overline{a s}$ - 'sit, 'sentar' \\
\hline$k s ̦ u d h$ - 'be hungry/tener hambre' & $k s ̦ a m$ - 'endure' 'resistir' \\
\hline bhuj- 'bend, curve/inclinarse, doblarse' & vas- 'put on, wear' 'vertir(se)' \\
\hline șrp- 'creep, slink, slither/deslizarse' & sac- 'accompany' 'acompañar' \\
\hline
\end{tabular}

7. Las pasivas comunes en sánscrito permiten que el agente sea expresado; pero en este caso, dice Klaiman (1991: 31) *namate dandah devadattena 'The stick bends by Devadatta'/La rama se inclinó por Devadata' como variante del ejemplo 2b, no es posible. 
En resumen, el tipo de sistemas de voz básica activa-media presenta los siguientes rasgos generales: (i) el lexicón verbal se organiza en tres clases de verbos (media tantum, activa tantum y alternantes). (ii) En la clase de verbos alternantes, que generalmente es la más amplia, la media tiene afinidad con la intransitividad (o detransitivización) y con diferentes clases de no-eventualidad (atélico, no puntual, futuro, etc.), aunque esto no implica que todas las construcciones medias en una lengua sean necesariamente intransitivas. (iii) Entre los verbos alternantes, la media implica la afectación del referente del sujeto lógico. Al respecto, codifica un rango de significados específicos que varía de lengua a lengua pero que coinciden en asignar al sujeto lógico el locus principal de los efectos de la acción denotada (Klaiman 1991: 105-8). Estos significados pueden ser: reflexividad, (auto)benefacción o malefacción, reciprocidad, cambio de estado espontáneo, pasividad, intensificación de la acción, orientación de la acción, entre otros. (iv) El grupo activa tantum expresa significados no deponentes, es decir, procesos y estados no controlados o no voluntariamente ejercidos por las entidades codificadas como sujeto; mientras que los verbos media tantum expresan significados deponentes ${ }^{8}$, es decir, acciones o disposiciones físicas o mentales que presuponen el control y la animacidad del sujeto lógico (Klaiman 1991:47).

En la próxima sección analizamos construcciones de voz media en toba, partiendo de la hipótesis de que esta lengua cuenta con un sistema de voz básica activa-media. Empezaremos mostrando cómo se organiza el lexicón verbal a partir de las marcas de voz para luego caracterizar distintas alternancias de la transitividad que ocurren junto a verbos medios alternantes $(\S 3.2 .1)$.

8. Klaiman (1991:97) toma el término 'deponente' (deponent) de Wheelock (1963:161) quien lo define etimológicamente a partir de la construcción latina de dē pōnō 'lay aside' -dejar de lado-. Es decir, se considera deponente el verbo cuya forma es pasiva pero que tiene significado activo y, en consecuencia, "deja de lado" su significado pasivo. Estos verbos ocurrían invariablemente con la conjugación $\underline{r}$ en latín. Tradicionalmente se los caracterizaba como no activos en la forma pero con significado activo; por ejemplo: hort'urge/ desear, ansiar'; pati- 'suffer/sufrir', experi- 'try/intentar', fate- 'confess/confesarse', sequ- 'follow/seguir', nasc- 'nacer'. 


\section{Análisis de construcciones de voz media y alternancias de la transitividad en Toba}

\subsection{Lexicón verbal y patrones de voz}

Con base en la compatibilidad del paradigma verbal $n$ - con 185 raíces verbales, diferenciamos tres clases: (i) activas, es decir, que no admiten el paradigma $n$-, como rke?e 'come', ro?oche 'duerme', Øhek 'se va', ilew 'se muere', entre otras; (ii) medias que sólo admiten el paradigma $n$ - como nachel 'se baña', ne?epe 'caza', entre otras y (iii) una gran mayoría de verbos alternantes?

En (1a) se ilustra una raíz intransitiva activa no causativa rkopchi 'se desmaya' que al causativizarse mantiene una marca de voz activa (ikopchaqchit 'hace desmayarse'). Los prefijos de 3ra., singular $r$ - e $i$ son seleccionados en función del grado de (in)transitividad de la raíz o base. No hemos documentado la forma media junto a esta raíz (1b).
(1a) r-kopchi so nogot, i-kopch-aqchit ñi n-ki?i ${ }^{10}$ 3VA-desmayarse DEM niño 3VA-desmayarse-CAU DEM 3POS- espíritu $^{11}$

9. En la muestra analizada el porcentaje de raíces alternantes es del 39,45\%, las raíces activas invariantes representan el $35,6 \%$ y las raíces medias invariantes el $23,78 \%$. Finalmente, un pequeño porcentaje $(1,08 \%)$, denominado "raíces supletivas", involucra dos raíces cuyo comportamiento es irregular en el sentido de que una de ellas (ñi?i 'se asusta, teme') completa su paradigma de conjugación o alterna con formas pacientivas y medias en la 1ra. y 2da. persona del plural según el Vocabulario Toba (Buckwalter y Buckwalter 2004); la segunda, constituye una forma defectiva que sólo se conjuga en 3ra. persona singular y plural (ñigi 'se cambia en'). En cuanto a la raíz ñi?i 'se asusta, teme' no hemos podido reconstruir mediante elicitación un paradigma de conjugación pacientivo; los hablantes consultados lo conjugan de acuerdo con el paradigma de voz media (Zurlo 2016a, b).

10. Los fonemas consonánticos y vocálicos del toba son (según Censabella 2002): $p, t, r$ $[d], t f, \int, k, g, h, q$, G, $, s, m, n, \eta, l, \lambda, 3, w, y, i, e, a, o$. En los ejemplos hemos optado por transcribir los símbolos $t f, \int, \lambda$ y $\eta$ como $c h, s h, \tilde{n}$ y $l l$, respectivamente.

11. Las abreviaturas y símbolos utilizados en la glosa para toba: $1=$ primera persona; $2=$ segunda persona; $3=$ tercera persona; $1 \mathrm{PL}=1$ ra persona plural; $\mathrm{A}=$ participante más parecido al agente en cláusulas transitivas; $\mathrm{AB}=$ direccional 'hacia abajo, abajo'; $\mathrm{ACER}=$ aplicativo 'acercamiento'; $\mathrm{AD}=$ aplicativo 'dentro'; $\mathrm{AGUA}=$ direccional 'hacia el agua'; $\mathrm{AL}=$ aplicativo alativo; $\mathrm{ALEJ}=$ aplicativo 'alejamiento'; $\mathrm{AMBITR}=$ ambitransitivo; $\mathrm{ANTIP}=$ antipasiva; $\mathrm{APL}=$ aplicativo; $\mathrm{ARR}=$ direccional 'hacia arriba'; $\mathrm{BEN}=$ aplicativo benefactivo; $\mathrm{COL}=$ colectivo; $\mathrm{COM}=$ aplicativo comitativo; CONT=aspecto imperfectivo continuo; CUANT=cuantificador; $D E M=$ demostrativo; $\mathrm{DEB}=$ aplicativo 'debajo'; DES= sufijo de modo 'desiderativo'; $\mathrm{DIM}=$ diminutivo; $\mathrm{DU}=$ dual; $\mathrm{ENF}=$ enfático; $\mathrm{EX}=$ existencial, presentativo; $\mathrm{EX} . \mathrm{NEG}=$ existencial, presentativo negativo; 
‘El espíritu le desmayó al niño’VT21 12

[Lit.: 'se desmaya el niño, el espíritu lo hizo desmayarse']

(1b) *nkopchi

En (2a) se ilustra la raíz intransitiva media invariante napilot 'se lava la cara'. También aquí el prefijo verbal $n$ - conforma una amalgama de persona, número y voz. No hemos registrado ninguna combinación de esta raíz con las marcas de 3ra. persona de voz activa (2b).
(2a) n-apilot
so nogotolek
3vM-lavarse.la.cara DEM niño.DIM.M
'Se lava la cara el niño'
(2b) *yapilot/*rapilot /*øapilot /*lapilot /*wapilot/*tapilot

Entre las raíces alternantes ilustradas en los ejemplos de (3) a (6) observamos dos patrones distintos de alternancias.

Un primer patrón involucra raíces verbales capaces de alternar sus marcas de voz sin morfología verbal adicional (3 a 5). En la mayoría de los casos, la alternancia involucra una raíz que es transitiva en voz activa e intransitiva en voz media, como en iyo 'lava algo'/ nyo 'se lava' (3); o bien, una raíz que es transitiva en voz activa y también en voz media, como en (4) yawan 've, conoce algo' / nawan 'vigila, espera (algo, alguien)'.
(3a) i-yo
(3b) n-yo
na
3vM-limpiar DEM
'El niño se limpia'
nogotolek
niño. DIM.M

EXT=direccional 'hacia el exterior'; $\mathrm{F}=$ femenino; IMP=impersonal; INT=direccional 'hacia un lugar cerrado, en el interior de'; INTENS=intensificador; INTR=intransitivo; IT=iterativo; LOC=locativo; $\mathrm{M}=$ masculino; $\mathrm{NEG}=$ negación; $\mathrm{NOMLZ}=$ nominalizador; $\mathrm{P} 1=$ =pronombre personal (1ra persona); PL.P.APL=plural argumento $\mathrm{P}$ aplicado; $\mathrm{IPOS}=$ prefijo posesivo inalienable; $\mathrm{POS}=$ prefijo posesivo alienable; $\mathrm{PDEM}=$ pronombre demostrativo; $\mathrm{PROG}=$ progresivo; RECEP=receptor; $\mathrm{RECP}=$ recíproco; $\mathrm{REFL}=$ reflexivo; $\mathrm{SOB}=$ aplicativo 'encima de, sobre'; $\mathrm{TR}=$ transitivo; $\mathrm{VA}=\mathrm{VOz}$ activa; $\mathrm{VM}=\mathrm{voz}$ media.

12. La sigla VT corresponde a las iniciales del Vocabulario toba de Buckwalter y Buckwalter [2004 (1980)], seguido del número de página. 
(4) yawan '(él/ella) conoce, ve (algo)'/ nawan '(él/ella) vigila, espera (algo, alguien)'
(4a) ñi piogonaq y-awan na n?araganaq DEM curandero 3VA-ver.conocer DEM víbora
'El curandero conoce la víbora para curar su picadura'
(Lit.: 'El curandero vió/conoce la víbora')
(4b) ?am n-awan ñi piogonaq
P2 3VM-ver.conocer DEM curandero
'Te vigila el curandero'

Con menor frecuencia, la alternancia involucra una raíz intransitiva en voz activa y también en voz media, como en (5), donde la alternancia se da entre la ausencia de prefijo personal (Ø) en la voz activa y el prefijo personal de voz media $(n-)$.

(5) ØparenaGan '(él/ella) salta'/ nparenaGan '(él/ella) salta (con ganas, de repente)'
(5a) Øparenagan so nogotolek 3va.saltar DEM niño. DIM.M 'Salta el niño'
(5b) ramaze n-parenagan P3 3vm-saltar 'Tiene ganas de saltar (él)'RM-dic

Un segundo patrón involucra raíces verbales alternantes que ocurren con marca media cuando se adiciona algún sufijo verbal como ser: el sufijo recíproco $(-a ? t)$, el reflexivo (-la?t), varios aplicativos y sufijos direccionales. La serie de ejemplos de (6) a (10) nos permite ilustrar este segundo subpatrón, en el cual no alternan las raíces desnudas (ilo 'mira/*nlo), sino los sintagmas verbales en los que la voz media coocurre junto a otros morfemas verbales.
(6a) i-lo
(6b) $*$ nlo
3VA-mirar
‘(Él/ella) mira’VT41 
(7a) *ilowek

(7b) n-lo-wek

3vM-mirar-eXT

'(él/ella) se despierta' VT41

[Lit.:'mira hacia afuera']

En $(8 \mathrm{a} / 8 \mathrm{~b})$ vemos un sintagma verbal alternante, conformado por la raíz - $l o$ 'mirar' más el sufijo aspectual continuo - $t a-+$ el sufijo verbal aplicativo -igi 'adentro' 13 .
(8a) i-lo-chigi
3VA-mirar-CONT.AD
(8b) n-lo-chigi
3 VM-mirar-CONT.AD
'Lo mira bien, se fija bien en' VT41
'Lo comprende' vT 135
['mira en lo profundo de' RM-dic]

La misma raíz verbal - lo 'mirar' con el índice pronominal de voz media coocurre con el sufijo recíproco en (9b) y con el sufijo reflexivo en (10b); en ambos casos falta la contraparte activa $(*$ ilotaa?t/*ilotapegela?t, respectivamente).
(9a) $*$ ilotaa?t ${ }^{14}$
(9b) n-lo-ta-a?t
3VM-mirar-CONT-RECP
'Se miran entre sí'
$(10 \mathrm{a}) *$ ilotapegela?t
(10b) n-lo-ta-pege-la?t
3VM-mirar-CONT-IT-REFL
'Se está mirando a sí misma, buscándose sola como piojos'
RM-dic (acicalamiento)

\footnotetext{
13. En el segmento conformado por el morfema -ta- 'imperfectivo continuo' + el morfema -igi interpretamos que se produce una palatalización regresiva por la presencia vocal /i/, cuyo resultado es -taigi $>$ chigi.

14. No se registra la combinatoria ilotaa?t, pero sí hallamos los siguientes sintagmas verbales de voz activa aplicativizados mediante el morfema - $20 t$ 'debajo de', cuyo significado es próximo al del sintagma medio recíproco de $(9 \mathrm{~b})$. Se trata de los sintagmas: ilo?ot ' le mira' VT41, iloto?ot 'le está mirando' y también ilotot 'le mira fijamente'. Mediante elicitación, registramos el sintagma verbal plural:

qomi se-lo-q-to-ot

P1PL 1PL.VA-mirar-1PL-CONT-DEB

'nos miramos (fijamente, ceja con ceja, como en un juego)'
} 
Cuando la lengua permite la conjugación en primera persona del plural ${ }^{15}$, comprobamos que se trata de la misma raíz verbal - $l o$ 'mirar', encerrada entre los circunfijos de 1ra. persona del plural, como se observa en (11), (12) y (13), respectivamente.
(11) in-lo-Go-wek
sowagat
1Reraga
1PL.VM-mirar-1 PL-EXT
COORD
rayo, fuego
'Nos despertamos a causa del rayo'
(12) ñi-lo-q-chi-gi Renawak na qom
1PL.VM-mirar-1PL-CONT-AD CUANT DEM gente
'Comprendemos a toda la gente'
(13) qomi in-lo-q-ta-a?t
P1PL 1PL.VM-mirar-1PL-CONT-RECP
'Nosotros nos miramos entre nosotros'

En síntesis, esta lengua manifiesta comportamientos característicos de un sistema de voz básica activa-media. Es decir, a semejanza del griego clásico, el lexicón verbal toba presenta tres clases mayoritarias (verbos activos invariantes, medios invariantes y alternantes). El grupo de verbos medios invariantes semánticamente expresa deponencia, o sea, comportamientos y disposiciones físicas o mentales que presuponen el control o la animacidad de S/A (natamen 'reza', nePepe 'marisca', nqat 'cosecha (algo)', nmit 'observa, reflexiona, juzga (algo)'). En contraste, el grupo de activos invariantes semánticamente expresa acciones y procesos físicos o naturales que no requieren el control o la animacidad de S/A (rket 'explota', ro?oche 'duerme', ilew 'muere', yawik' 'arde') ${ }^{16}$. El eje que articula este sistema de voz básica activo/

15. En las distintas sesiones de elicitación no pudimos constatar que el reflexivo ocurra con formas de plural, pues en ese tramo funcional ocurre el morfema de recíproco cuando el sujeto está pluralizado. La estrategia de conjugar en 1 ra persona del plural $(\tilde{n}-\ldots-q)$, permite comprobar si el segmento encerrado entre los circunfijos de 1ra persona corresponde a la misma raíz (-achel) o a una forma verbal derivada, por ejemplo, la base causativizada (-achelaGan), como se puede ver en (i) y (ii), respectivamente.
(i) ñ-achel-aq
1PL.VM-bañarse-1PL
(ii) ñ-achelaGan-aq
'Nos bañamos'
1PL.VM-bañarse.CAU-1PL
'Nosotros bañamos a' 
medio está dado por el grupo mayoritario de verbos alternantes. Al respecto, la alternancia documentada con mayor frecuencia en la muestra analizada involucra especialmente el índice pronominal de 3ra persona $i$-[y-], el cual, típicamente ocurre junto a raíces y bases activas transitivas. En oposición, la raíz media alternante, prefijada con $n$ - en la 3ra persona, se mantiene transitiva ${ }^{17}$ (4), intransitiva (5) o manifiesta detransitivización (3). Es decir, la alternancia entre el verbo en voz activa, prefijado con $i$ - $[y$ - $]$ y el verbo en voz media, prefijado con $n$ - aunque manifiesta afinidad con detransitivización no tiene una única resolución.

Para Klaiman (1991:105) el significado central o la función diferencial de la voz media en el grupo de verbos alternantes puede caracterizarse como afectación de S/A y subsume una variedad de significados como: reflexividad, autobenefacción, cambio de estado, entre otros.

A continuación definimos de manera general algunas alternancias de la transitividad y luego presentamos algunas de las que ocurren junto a verbos medios alternantes; comenzamos con las que típicamente disminuyen la transitividad de la cláusula (media reflexiva, media recíproca) y finalizamos con las que aumentan o reorganizan el número y tipo de participantes centrales (aplicativos).

que la voz activa cuenta con una escisión en la marcación de la 3ra persona (tabla 1). Es necesario señalar que las motivaciones semántico-sintácticas y léxicas de estas marcas diferenciales de A y S para la 3ra persona, voz activa, en toba todavía no han sido explicadas íntegramente.

17. En el primer caso de alternancia activa transitiva y media transitiva, se mantiene la estructura argumental de la raíz verbal y los cambios acarreados por la marca de voz son de índole semántica, especialmente vinculados con la orientación de los participantes en el proceso/acción y con el mayor involucramiento y/o afectación del protagonista en la acción denotada. Este tipo de alternancias se observa junto a verbos transitivos o ditransitivos que involucran traslación, transferencia y movimiento de objetos, algunos incluyen cambio de posición o de lugar del objeto (yache 'lo lleva'/ nache 'lo trae'; yawek 'traslada algo hacia' / nawek 'traslada algo hacia'; iro 'mueve algo hacia'/nro 'mueve algo hacia', yamaq 'lo empuja, envía, manda hacia allá'/namaq 'lo empuja, envía, manda hacia acá', iwi? 'lo alcanza, llega allá'/nwi? 'lo alcanza, se llega acá'); percepción y cognición (yawan 'lo conoce, ve'/nawan 'lo vigila, espera'); comunicación y comportamiento social-cultural (iyagana 'lo llama'/nyagana 'lo llama'). 


\subsection{Alternancias de la transitividad y mecanismos de ajuste de valencia}

De acuerdo con Dixon y Aikhenvald (2000: 5), las lenguas pueden organizar los verbos según su grado de transitividad mediante distintos recursos: léxicos, morfológicos y sintácticos. Típicamente, estos recursos /mecanismos morfosintácticos permiten reducir o incrementar el número de argumentos centrales o bien, mantener el mismo número de argumentos pero alterar sus propiedades semánticas (Dixon y Aikhenvald 2000: 6). La tabla 3 representa de manera esquemática algunos mecanismos de cambio de valencia que prototípicamente se aplican sobre verbos transitivos para derivar verbos intransitivos, como ser la pasiva (en la cual el P original se convierte en S) y la antipasiva (donde A se convierte en $\mathrm{S}$ ) o sobre verbos intransitivos para derivar verbos transitivos como es el caso de las causativas (donde el $\mathrm{S}$ original se convierte en $\mathrm{P}$ ) y las aplicativas (en las cuales $\mathrm{S}$ se convierte en $\mathrm{A}$ ).

Tabla 3 - Mecanismos de cambios de valencia (Dixon y Aikhenvald 2000: 6)

\begin{tabular}{lll}
\hline $\begin{array}{l}\text { Prototípicamente } \\
\text { aplicada a raíz/base }\end{array}$ & $\begin{array}{c}\text { Reasignación argumental } \\
\text { (a) }\end{array}$ & $\begin{array}{c}\text { Reasignación argumental } \\
\text { (b) }\end{array}$ \\
$\begin{array}{l}\text { (i) TRANSITIVA } \\
\text { (ii) INTRANSITIVA }\end{array}$ & O se vuelve S (pasiva) & A se vuelve S (antipasiva) \\
\hline
\end{tabular}

En toba, las operaciones de modificación de la valencia son seis: (i) antipasiva, (ii) pasiva no promocional o impersonal, (iii) reflexivo y (iv) recíproco, (v) varias construcciones causativas morfológicas y (vi) varias construcciones aplicativas. En este artículo presentaremos algunos de estos mecanismos en función de su ocurrencia junto a verbos medios alternantes del corpus analizado.

Alternancias de la transitividad y voz media en toba: cuestiones generales

Teniendo en cuenta que nuestra propuesta de análisis plantea comportamientos de un sistema de voz básica activa-media en la 
lengua toba, no podemos agrupar las construcciones medias únicamente entre las operaciones detransitivizadoras (de disminución o fusión de participantes), ni tampoco generalizar que el marcador $n$ - funciona como un operador detransitivizador ya que estaríamos desatendiendo algunas particularidades morfosintácticas de esta lengua. La voz media en toba representa una categoría semántico-sintáctica compleja.

En primer lugar, encontramos en toba construcciones medias heterogéneas: un grupo de verbos medios alternantes (aquellos capaces de alternar sus marcas de voz) y un grupo de verbos medios invariantes (o media lexicalizada, es decir, verbos que jamás ocurren sin la marca media). Dentro del grupo de verbos medios alternantes también encontramos comportamientos heterogéneos, pues observamos que en la muestra analizada, el porcentaje de raíces alternantes medias con detransitivización (pérdida argumental) alcanza el 57\%. Este porcentaje nos sugiere por un lado, que existe afinidad entre la detransitivización y la voz media para verbos alternantes del toba; tendencia esperada según el estudio de Klaiman (1991). Por otro lado, también sugiere que hay cambios de valencia o alternancias de la transitividad que no involucran afijos verbales derivativos, sino que se realizan a través del cambio de índices pronominales ${ }^{18}$, como en (14) y (15). La alternancia en (14) involucra la raíz activa transitiva $i$-chigoq 'sacudir algo' (14a) que al ocurrir con voz media (14b) denota un cambio cumplido en el argumento único (locus de la afectación).

\begin{tabular}{|c|c|c|c|}
\hline 4a) (ramaze) & i-chigoq & na & nogo \\
\hline P3 & 3vA-sacudir & DEM & ropa \\
\hline
\end{tabular}

\footnotetext{
18. Es necesario señalar que este procedimiento es productivo y también opera sobre verbos activos, en los cuales el cambio del índice pronominal de 3ra persona de la voz activa origina "verbos distintos", a pesar de que tengan en común la misma raíz; por ejemplo: $y$-asot 'lo patea' (TR) / r-asot 'patea, baila' (INTR). Para Censabella (2008:107) opera sobre un grupo muy pequeño de raíces verbales intransitivas que admiten el pasaje de eventos espontáneos o agentivos a verbos causativos, mediante el cambio del personal verbal de 3ra persona activa, como en el par woko? 'se vuelca' / iko? 'lo vuelca'. Según la autora, este procedimiento estaría respondiendo a un estadío anterior de la lengua, en el que era más funcional un sistema de conjugación donde la selección del personal de tercera activa se realizaba bajo criterios semánticos (...) En la actualidad esta alternancia está totalmente lexicalizada (...) y los hablantes consideran que se trata de verbos distintos (Censabella 2008:108).
} 


$$
\begin{array}{llll}
\text { (14b) } & \text { n-chigoq } & \text { ñi } & \text { pioq } \\
\text { 3vM-sacudir } & \text { DEM } & \text { perro } \\
& \text { 'Se sacude el perro' } &
\end{array}
$$

También manifiestan detransitivización semántico-sintáctica verbos de comportamiento social como en (15).

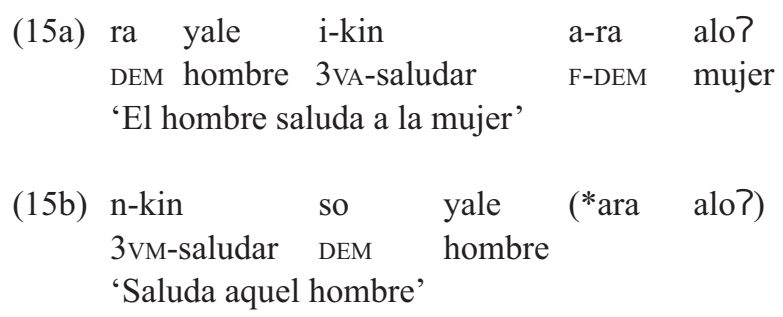

En estos casos $(14 b, 15 b)$, la construcción media alternante es intransitiva y expresa una acción que se desenvuelve -y cuyos resultados se acumulan- sobre un único argumento (S). Más que una construcción antipasiva prototípica ${ }^{19}$, aquí observamos un proceso de detransitivización o pérdida de diferenciación entre A y $\mathrm{P}$, pues el único participante es, a la vez, iniciador y locus de afectación.

Por último, en esta lengua las operaciones de cambio de valencia pueden ocurrir sobre raíces medias invariantes y/o alternantes. Las restricciones se vinculan con el grado de (in)transitividad de la raíz y las compatibilidades semánticas entre los morfemas de cambio de valencia y el contenido léxico semántico de la raíz verbal. Este punto es importante porque revela el impacto estructural de la marca de voz en el lexicón verbal toba.

19. En toba, la antipasiva prototípica $(r-\ldots-a G a n)$ : se construye a partir de una raíz activa transitiva, típicamente prefijada con $i$ - en la 3ra persona, la cual se intransitiviza mediante el morfema - $a$ Gan y, en consecuencia, toma el prefijo $r$-, de 3ra. persona. La antipasiva prototípica indica que la acción expresada se realiza de manera consciente y voluntaria por parte del sujeto-agente; además, disminuye la transitividad del verbo, pues desaparece $\mathrm{P}$ como se observa en el siguiente par de ejemplos (ramaze i-nat so Pedro 'Aquel pregunta a Pedro'[TR]/ ramaze r-nat-aGan 'Aquel pregunta' [INTR]. 


\subsubsection{Detransitivización y voz media}

\section{Construcciones de voz media y sufijo reflexivo (-la?t)}

El sufijo reflexivo -la?t sólo ocurre con voz media y es invariable; no indexa rasgos de número o de persona, que permitan definirlo como un marcador de correferencialidad ${ }^{20}$. Más bien, se comporta como un típico operador de cambio de valencia verbal ya que impide la posible expresión del mismo referente (parte/todo) y clausura la presencia de un participante animado y humano en la posición de P. En la muestra analizada, lo documentamos sólo en singular (16a), pero Buckwalter y Buckwalter (2004:344) muestran un uso en plural (16b).

(16a) ñi-shewga-la?t

1VM-hamacar-REFL

'Me hamaco a mí misma'

(16b) ñi-shewga-Ga-la?t

1 PL.VM-hamacar-1PL-REFL

'Nos hamacamos' vT344

Este sufijo reflexivo no es compatible con cualquier verbo medio. En general, no ocurre junto a predicados medios invariantes intransitivos, que expresan autobenefacción, cambio de estado/posición o acción sobre uno mismo (17). En estos casos, la voz media inherente es la estrategia empleada para expresar que los efectos de la acción recaen sobre el participante iniciador.

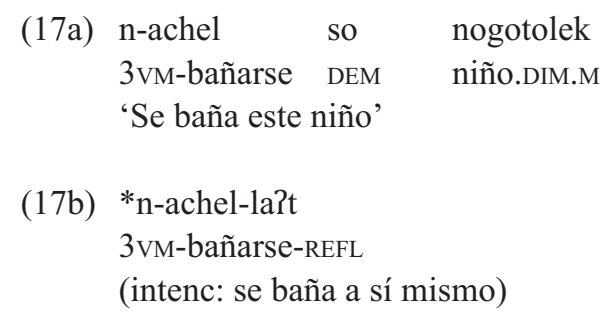

En (18) vemos el comportamiento de la raíz activa transitiva yalawat 'matar a' y en (18b) la base verbal media alternante intransitiva (nalaat 
'se mata, guerrea'), cuyo significado es distinto al de la construcción media reflexiva prototípica con el sufijo reflexivo -la?t (18c), en la cual la acción se dirige hacia el mismo participante iniciador.

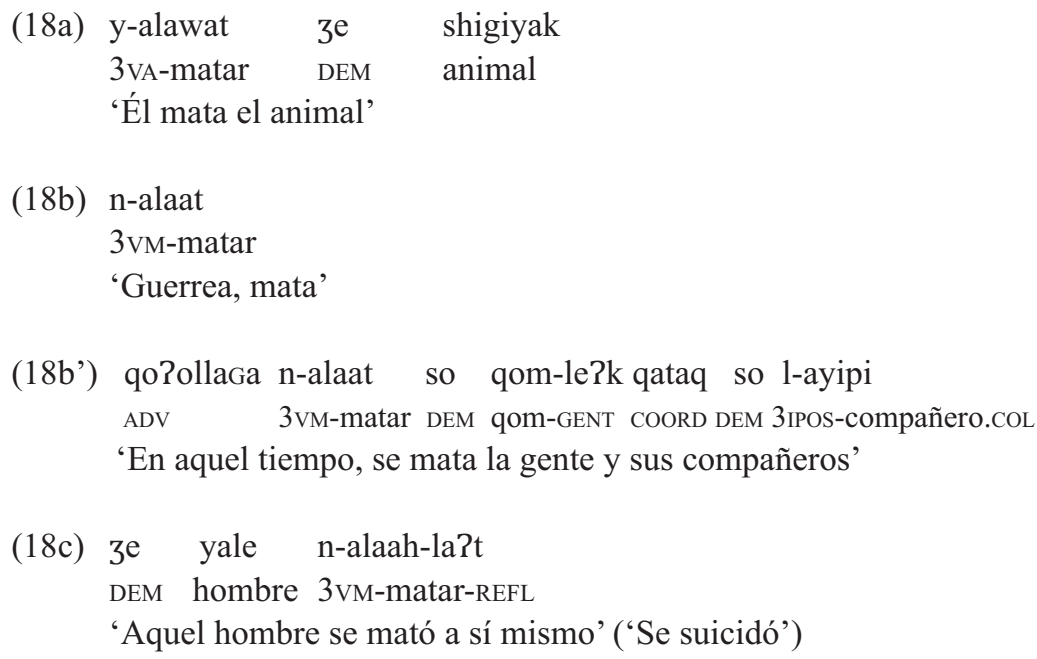

Los predicados medios que aceptan esta reflexivización mediante el sufijo - la?t son verbos transitivos que denotan procesos y actividades mentales (percepción, cognición y emoción interna) o comunicativas y que involucran participantes humanos o animados. Con menor frecuencia la reflexivización se aplica sobre verbos (di)transitivos y puede originar una construcción medio-reflexiva transitiva con cláusula completiva (19c, 20b, 20c).

En (19) ocurre una raíz verbal alternante de cognición y percepción, que en (19c) admite una cláusula completiva para predicar un contenido de conciencia (objeto comunicado, mensaje, pensamiento o percepción).
(19a) ayem mashe s-awan-a enawaq chaco P1 ADV 1VA-ver.conocer-APL:AL CUANT Chaco 'Yo ya conozco todo el Chaco'

(19b) Pam ñ-awan

P2 1VM- ver.conocer

'Te vigilo' RM-dic 
(19c) soche ñ-awaa-la?t

ra ayim qawemaik

ADV 1VM-ver.conocer-REFL DEM P1 maldad.NOMLZ

'Nomás (me) descubro en mí mismo que yo (soy) malo’ RM-dic

En (20) ocurre una raíz alternante que admite cláusula completiva para expresar el objeto comunicado, mensaje.

(20a) so shiyagawolek n-ta?a i-nat so nyomagaik DEM persona 3IPOS -jefe 3VA-preguntar DEM bebedor “...el Principito preguntó al bebedor que estaba sentado en silencio" (El Principito, 31). [Lit.: 'la personita jefe preguntó al bebedor']

(20b) maiche ñi-nat-tapig-ela?t eetek ka lo?ogoyagak na logo ENF 1VM-preguntar-CONT.IT-REFL ADV DEM color DEM ropa 'Dudo también sobre el color de su traje'. (El Principito, 13)

[Lit.: 'Yo nomás me pregunto a mí mismo cómo el color de su ropa']

(20c) in-nah-la?t eetek ra mashe ayem ze 1VM-preguntar-REFL ADV DEM ADV P1 maduro 'Me pregunto cómo seré de grande' RM-dic

Construcciones de voz media y sufijo recíproco (-a?t)

El morfema recíproco - $a$ ?t también es un sufijo invariable que se traduce como "entre sí/ entre ellos", "uno con otro", "uno y otro", "juntos". En trabajos anteriores describimos varios patrones de uso del morfema - $a$ ?t, el cual puede ocurrir sobre predicados (no sólo verbales ${ }^{21}$ ) capaces de codificar una relación simétrica, simultánea e idéntica entre dos o más participantes.

La construcción media admite el sufijo - $a$ ?t cuando los reciprocantes o participantes de la relación simétrica predicada son codificados en la misma función sintáctica: ya sea como $\mathrm{S}$

21. Este morfema puede ocurrir junto a predicados de tipo adverbial que expresan relaciones comparativas (eta?a?t 'son iguales entre sí'/ yotta?a?t 'son diferentes entre sí'). Entre las construcciones recíprocas del toba observamos los siguientes subtipos semánticos: (i) relación simétrica en un par/pareja; (ii) relación simétrica en grupo; (iii) cadenas de acciones y (iv) plural de relación simétrica de parentesco (yaqaya?t 'hermanos entre sí'). También se usa sobre neologismos, construidos a partir de préstamos del español (npelottega?t 'juegan pelota entre sí', lit.: ‘se están peloteando'). 
(en cláusulas intransitivas) o como $\mathrm{P}$ (en cláusulas transitivas), o incluso como A (en cláusulas transitivas). Estos patrones también se observan junto a raíces y bases verbales de voz activa, dependiendo del grado de transitividad de la raíz involucrada.

Así, en (21) observamos la raíz activa transitiva alternante iwaGan 'lo golpea, pega, pelea' (21a) que permite una construcción recíproca activa con P pluralizado (21b).

(21) iwaGan 'golpea, pega a (alguien, algo)' / nwaGan 'golpea, pelea'

$$
\begin{aligned}
& \text { (21a) ayimi-wagan so shiyagawa } \\
& \text { P1 3vA-golpear DEM persona } \\
& \text { 'Me pegó aquella persona' RM-dic }
\end{aligned}
$$

(21b) ?a-wagan-a?t na ar-wagazi na l-ya qaltaq na l-ya 2VA-golpear-RECP DEM 2IPOS-mano.PL DEM 3IPOS-otro COORD DEM 3IPOS-otro 'Golpea tus manos una contra otra'(El Principito, 29)

(Lit: golpeá entre sí tus manos, una y la otra)

En (22a) se observa la forma media alternante nwaGan 'golpea, pelea, se pelea' y las construcciones medias recíprocas intransitivas con S plural o coordinado (22b, 22c).

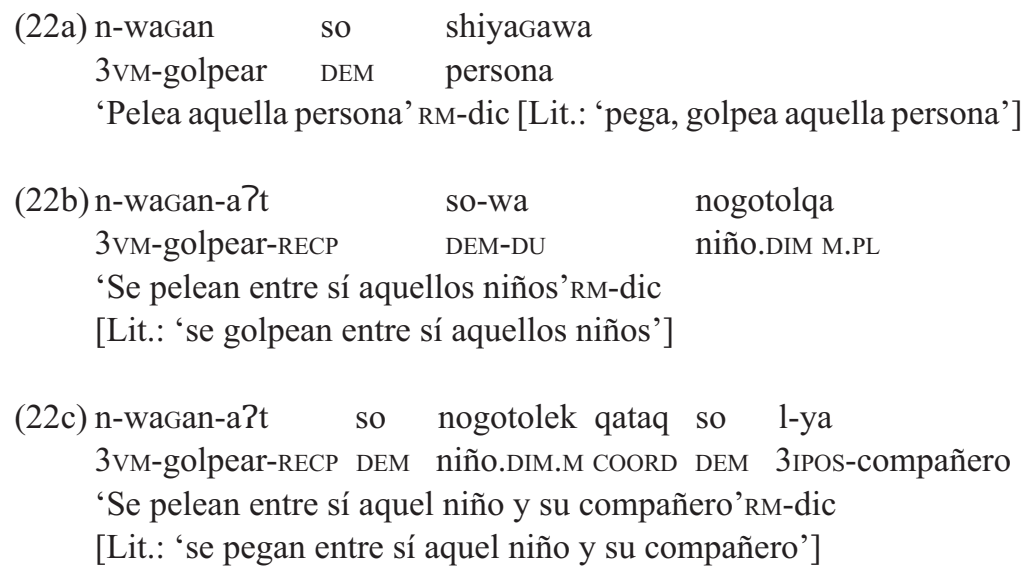

Con menor frecuencia, se observan cláusulas transitivas medias en las cuales los reciprocantes se codifican en la posición de A 
(pluralizado), como se observa en (23b) junto al verbo transitivo alternante -kozigen 'avisa, despide (a)'.

(23a) se-kozigen-aq na qompi, cha?azi qolaq-tak

1PL.vA-avisar-1PL DEM qom.COL COORD 1PL.VA.ir.1PL-PROG

'Avisamos a la gente porque nos vamos'

(23b) mashe in-kozigen-aG-a?t ke?eka laponagak

ADV 1PL.VM-avisar-1PL-RECP PDEM reunión

'Ya nos avisamos -entre nosotros- esa reunión'

\subsubsection{Transitivización y voz media en toba}

\section{Construcciones de voz media y sufijos aplicativos}

Los comportamientos observados con mayor frecuencia involucran una raíz verbal media intransitiva que al aplicativizarse se convierte en transitiva. Es decir, sobre cláusulas intransitivas medias alternantes como en (24), el aplicativo permite formar cláusulas transitivas con un argumento (periférico) promovido al espacio funcional de $\mathrm{P}$.

En (24) la raíz media alternante intransitiva nalaat 'se mata' (24b) permite incorporar un participante adicional (el compañero o socio de la acción) mediante el sufijo aplicativo comitativo -e? (24c).

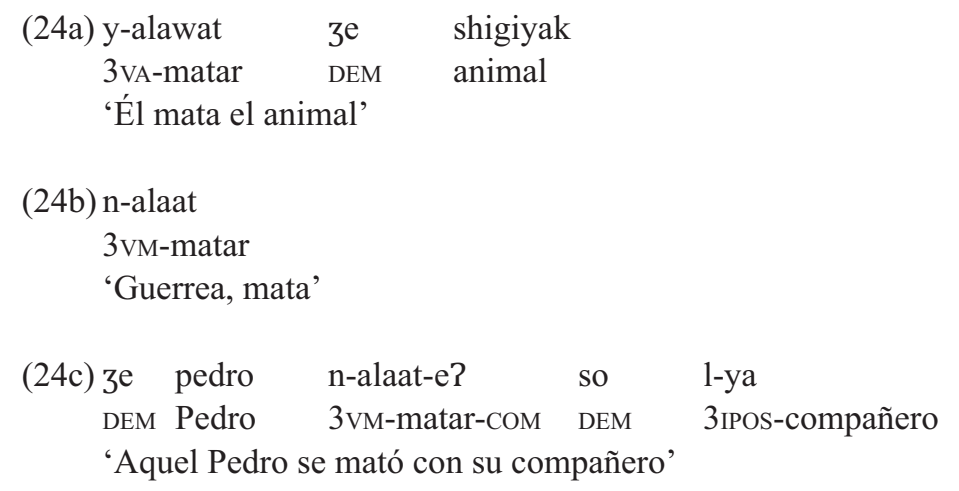

En otros casos, el aplicativo no aumenta la valencia verbal, sino que reconfigura la relación entre A y $\mathrm{P}$ al permitir la expresión de un participante periférico en la posición central de $\mathrm{P}$ (26). 
En (25) la raíz media alternante transitiva -men 'canjea, intercambia (para sí)', con el sufijo aplicativo -get permite expresar el objeto intercambiado, nmenaget 'canjea algo por, remplaza a', en (25c). Esta raíz media aplicativizada permite expresar nuevos significados como en $(25 d)$.

(25) imen 'canjea, vende, intercambia' / nmen 'canjea, compra, intercambia'

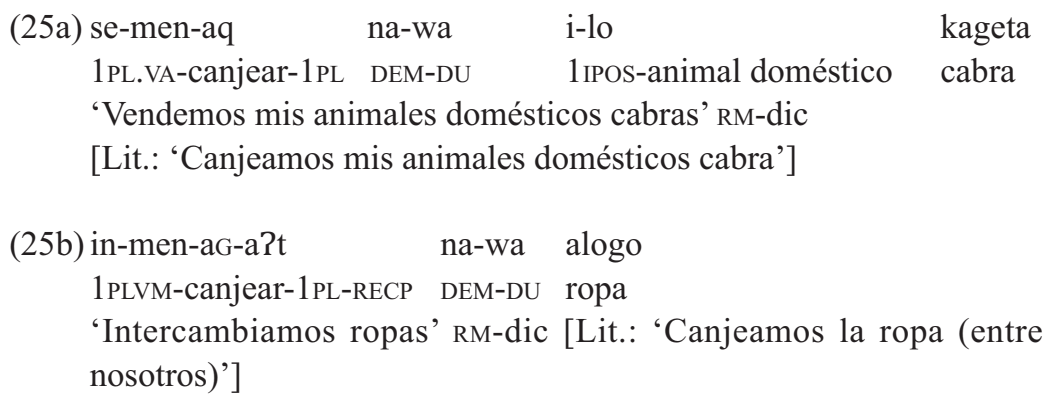

Con menor frecuencia, un sufijo aplicativo puede incrementar la valencia de un verbo transitivo (26a) conviertiéndolo en ditransitivo (27).

(26) iro 'mueve algo hacia allá, traslada algo'/ nro 'mueve algo hacia acá, traslada algo'

$\begin{array}{ccl}\text { (26a) Pa-ro-wo } & \text { na } & \text { qoipaq } \\ \text { 2vA-trasladar-INT } & \text { DEM } & \text { leña } \\ \text { ‘Llevá adentro esa leña!' } & \\ & & \\ \text { (26b) Pan-ro-wo } & \text { ka } & \text { qan-qoipaq! } \\ \text { 2vM-trasladar-INT } & \text { DEM } & \text { 1PL.POS-leña } \\ \text { ¡Traé (adentro) nuestra leña!' } & \end{array}$


En (26) se presenta la raíz alternante transitiva -ro 'mover algo hacia', que se transforma en el verbo $n r a ? a$ 'se lo mueve hacia, se lo trae'(28) junto al aplicativo alativo - $a$ ?. Aquí el argumento A es codificado mediante el morfema discontinuo de 1ra. p. plural voz media $(\tilde{n}-\ldots-q)$, que se corresponde con un rol semántico agente; el participante $\mathrm{P}$ es codificado mediante la frase nominal pospuesta al verbo ( $r a$ n?aqtak 'la palabra, el evangelio'), se corresponde con el tema y un tercer participante es codificado mediante el pronombre libre antepuesto al verbo qami 'ustedes'. Este participante receptor es introducido mediante el sufijo aplicativo alativo ( $-2 a$ 'hacia') y al ser un referente plural exige el sufijo pluralizador de objeto aplicado (-lo) inmediatamente pospuesto.

\section{Conclusiones}

En este trabajo hemos analizado construcciones de voz media de la lengua toba y, en particular, describimos algunas alternancias de la transitividad que ocurren junto a verbos medios alternantes.

En primer lugar, observamos que la voz media en toba se codifica a través del paradigma verbal $n$-. Argumentamos que esta lengua exhibe características de un sistema de voz básica activa-media (Klaiman 1991), ya que organiza el lexicón verbal en tres grandes clases: (a) verbos que no admiten el paradigma $n$ - como rke?e 'come', ro?oche 'duerme', Øhek 'se va', entre otros; (b) verbos que sólo admiten el paradigma $n$ - (y que, en este sentido constituyen una media lexicalizada) como nachel 'se baña', ne?epe 'caza', entre otros y (c) un grupo mayoritario de verbos alternantes como yapaGagen 'educar a, enseñar (algo)'/napaGagen 'educarse, aprender (algo)', yawek 'lo traslada, lo lleva'/ nawek 'lo traslada hacia acá, lo trae', ilo 'mira'/ nlowek 'se despierta', entre otros.

En segundo lugar, señalamos que la voz media es una categoría semántica compleja que abarca distintos tipos de situaciones (Kemmer 
1993) y comprende construcciones heterogéneas (media léxica o invariante y media morfológica o alternante), nucleadas en torno al significado prototípico de afectación de $S / A$, es decir, cuando los efectos de la acción recaen sobre el participante iniciador (iyo 'lava (algo)/ nyo 'se lava').

Finalmente, nos preguntamos cómo resolver el desafío de analizar las alternancias de la transitividad en este tipo de sistemas de voz básica. Al respecto, observamos que la marca media tiene afinidad con detransitivización o pérdida argumental (construcción media alternante de cambio de estado y afectación de S), aunque hallamos patrones de alternancia donde no se altera la (in)transitividad de la raíz (yawan 've, conoce algo' - TR/nawan 'vigila, espera algo, alguien'-TR) $\emptyset$ parenaGan 'salta' INTR/ nparenaGan 'salta con ganas, de repente' INTR). Por otro lado, describimos las alternancias de la transitividad desencadenadas por morfemas verbales como recíproco, reflexivo y aplicativos; de los que sólo el sufijo verbal -la?t exige la ocurrencia de voz media. Con relación al complejo sistema de voz básica activamedia de esta lengua han quedado varios temas pendientes, por ejemplo, caracterizar las alternancias de la transitividad observadas entre las raíces y bases verbales invariantes de voz activa y media, especialmente mostrar los mecanismos de transitivización (causativas morfológicas) y de detransitivización (pasiva no promocional o impersonal) cuya frecuencia es más significativa junto a raíces verbales medias invariantes, dentro del corpus analizado. El panorama de las alternancias de la transitividad junto a estas raíces verbales permitiría enriquecer la comprensión teórica en torno a los sistemas de voz básica $\mathrm{y}$, en particular, aportar a la comprensión de esta lengua.

\section{Referencias bibliograficas}

BENVENISTE, Emile. 1966 [1950]. "Actif et moyen dans le verbe", en Problèmes de linguistique générale. Paris: Gallimard. p. 168-175.

BIGOT, Margot. 1994. "La lengua Qom (toba) del Chaco, Argentina (Expresión del espacio en los lexemas verbales)", en A. Fernández Garay y P. Viegas Barros, Actas de las Segundas Jornadas de Lingüística Aborigen. Buenos Aires: Universidad de Buenos Aires, Facultad de Filosofía y Letras, Instituto de Lingüística. p. 177-189. 
BUCKWALTER, Albert y BUCKWALTER, Louis. 2004 [1980]. Vocabulario toba seguido de algunos apuntes sobre la gramática del idioma toba. Primera edición revisada. 3ra reimpresión. Equipo Menonita, Formosa, Argentina.

CARPIO, María Belén. 2007a. Sistemas de alineación en toba (familia guaycurú, Argentina). Tesis de Maestría. Universidad de Sonora. Hermosillo, México.

. 2007b. Número y categorías afines en la lengua toba. En A. Fernández Garay, et al. (Eds.), Estudios lingüísticos y sociolingüísticos de lenguas indigenas sudamericanas (pp: 13-27). Santa Rosa: Universidad Nacional de La Pampa.

2012. Fonología y morfosintaxis de la lengua hablada por grupos tobas en el oeste de Formosa (Argentina). München: LINCOM.

CARPIO, Maria Belén y CENSABELLA, Marisa. 2010. Género y número en toba (Flia. guaycurú, Argentina). En Z. E. Fernández, Z. y R. Arzápalo Marín (Eds.), Estudios de lenguas amerindias 2: contribuciones al estudio de las lenguas originarias de América. Hermosillo: UNISON. p. 57-84.

CENSABELLA, Marisa. 1998. "Axiología de la voz media en toba", en P. Viegas Barros y M. Censabella (Eds.), Actas III Jornadas de Lingüística Aborigen. Buenos Aires: Instituto de Lingüística, UBA, pp. 91-101.

. 2002. Descripción funcional de un corpus en lengua toba (familia Guaycurú, Argentina). Sistema fonológico, clases sintácticas y derivación. Aspectos de sincronía dinámica. Tesis Doctoral. Universidad Nacional de Córdoba.

2007. Los aplicativos locativo y alativo en toba. En A. Fernández Garay y M. Malvestitti (Eds.), Estudios Lingüísticos y sociolingüísticos de lenguas indígenas sudamericanas (pp. 29-50). Santa Rosa: Universidad Nacional de La Pampa.

2008. "Derivación causativa en lengua toba", en C. Chamoreau, Z. E. Fernandez, S. Wichmann y A. Gonzalez (Eds.), Studies in voice and transitivity (Estudios de voz y transitividad). Munich: Lincom. p. 105-125.

COMRIE, Bernard. 1978. "Ergativity”, en W. P. Lehmann (Ed.), Syntactic Typology: Studies in the Phenomenology of Language. Texas: University of Texas Press. p. 329-394.

DGEEC. 2013. III Censo Nacional de Población y Viviendas para Pueblos Indígenas. Pueblos indígenas en el Paraguay. Resultados preliminares 2012. Asunción: Dirección General de Estadísticas, Encuestas y Censos. 
DIXON, Robert M. W. y Alexandra Y. AIKHENVALD. 2000. Changing valency. Case studies in transitivity. Cambridge University Press.

GIVÓN, Talmy. 2001. Syntax. A functional-typological introduction. Vols. I y II. Amsterdam/Philadelphia: John Benjamins.

GONZÁLEZ, Raúl. 2013. "Índices pronominales en cláusulas con argumentos aplicados en toba (familia guaycurú)", en M. Censabella y C. Messineo (Eds.), Lenguas indígenas de América del Sur II. Morfosintáxis y contacto de lenguas. Volúmenes temáticos de la Sociedad Argentina de Lingüística (SAL.) Mendoza: Universidad Nacional de Cuyo. p. 59-72.

. 2015. Estudio fonológico y morfosintáctico de la lengua toba hablada en el este de la provincia de Formosa (Argentina). München: LINCOM.

GRONDONA, Verónica. 1998. A Grammar of Mocovi. Tesis Dotoral. Pittsburgh: University of Pittsburgh.

GUALDIERI, Beatriz. 1998. Mocovi (Guaycuru) Fonologia e morfossintaxe. Tesis Doctoral. Campinas: Universidade Estadual de Campinas.

INDEC. 2012. Censo Nacional de Población, Hogares y Viviendas 2010: Censo del Bicentenario: Resultados definitivos, Serie B N ${ }^{\circ} 2$. Buenos Aires. Disponible en: http://www.censo2010.indec.gov.ar/archivos/ censo2010_tomo1.pdf

KEMMER, Suzanne 1993. The middle voice (Typological Studies in Language, 23). Amsterdam/Philadelphia: John Benjamins.

KLAIMAN, Miriam H. 1991. Grammatical Voice. Cambridge University Press.

KLEIN, Harriet. M. 1981. Una gramática de la lengua toba: morfología verbal y nominal. Tesis Doctoral. Universidad de la República, Montevideo.

MESSINEO, Cristina. 2002. La marcación verbal activa/inactiva en toba (guaycurú) y sus motivaciones, Liames, 2: 49-62. 2003. Lengua toba (guaycurú). Aspectos gramaticales y discursivos. München: LINCOM.

SHIBATANI, Masayoshi y Ketut Artawa. 2007. The middle voice in Balinese. XIII ${ }^{\text {th }}$ South East Asian Linguistics Society (SEALS) Conference at UCLA.

SAINT-EXUPERY, Antoine de. 2005. So Shiỹaxauolec Nta'a (Le Petit Prince). Buenos Aires: Association pour l'Echange Artistique et Culturel (AEAC).

VIDAL, Alejandra. 2001. Pilagá Grammar (Guaykuruan Family, Argentina). Tesis Doctoral Universidad de Oregon. 
WHEELOCK, Frederic M. 1963. Latin: an introductory course based on ancient authors. 3rd. edn. New York: Barnes \& Noble.

ZURLO, Adriana. 2011. La expresión del número verbal en toba. En A. Fernández Garay y A. Díaz Fernández (Eds.), Investigaciones sobre lenguas indígenas sudamericanas (pp. 315-341). Santa Rosa: Universidad Nacional de La Pampa.

. 2014. Simetría, reciprocidad y más: usos del morfema toba -a?t. En M. Malvesttiti et al. Actas del III Encuentro de Lenguas Indígenas Americanas (ELIA) (pp. 649-661). Bariloche: Universidad Nacional de Río Negro. Disponible en: http://www.unrn.edu.ar/publicaciones/ index.php/35-contenidos/libros/77-qactas-lenguaq.

. 2016a. Sistema medio en dos lenguas de Resistencia: toba y español. Estudio tipológico funcional. (Tesis doctoral). Resistencia (Argentina): Universidad Nacional del Nordeste.

. 2016b. "Voz media en Toba (Guaycurú)". Liames, 16, N. 2 (2016): 285-306. URL: https://periodicos.sbu.unicamp.br/ojs/index. php/liames/article/view/8646309.

Recebido em: 17/07/2018

Aprovado em: 16/01/2019

\section{Nota de agradecimiento:}

Quiero agradecer el apoyo financiero brindado por el Consejo Nacional de Investigaciones Científicas y Técnicas de la República Argentina (CONICET) y por la Universidad Nacional del Nordeste (UNNE). Asimismo, agradezco a los evaluadores anónimos, cuyos comentarios y sugerencias permitieron mejorar muchos aspectos de este artículo. 